\title{
Ion Pairing Controls Physical Properties of Ionic Liquid-Solvent Mixtures
}

\author{
Ray Matsumoto, ${ }^{\dagger, \ddagger}$ Matthew W. Thompson, ${ }^{\dagger, \ddagger}$ and Peter T. Cummings, ${ }^{*, \dagger}, \ddagger$ \\ $\dagger$ Department of Chemical and Biomolecular Engineering, Vanderbilt University, Nashville, \\ TN, USA \\ $\ddagger$ Multiscale Modeling and Simulation Center, Vanderbilt University, Nashville, TN, USA \\ E-mail: peter.cummings@vanderbilt.edu
}

\section{Liquid Densities}

The liquid densities of the ionic liquid - organic solvents mixtures were calculated as a function of composition. The table below (Fig. S1) displays the densities of the mixtures at various compositions in terms of mass fraction.

\section{Computation of Ion Correlation Lifetimes}

The calculation of ion pairing and caging lifetimes were discussed briefly in the main text. This is done so by computing the number of ion pairs in the first frame, and then computing which of these pairs remains in the subsequent pairs. The distance criteria use to calculate pairs was determined by the first well displayed in the radial distribution functions (RDFs) between $\left[B M I M^{+}\right]$and $\left[T f_{2} N^{-}\right]$. Example RDFs are shown in Fig. S2. The ratio of remaining pairs is plotted against time, and the curve is fitted with a stretch expoential function get the pairing/caging lifetimes. These plots are shown in Fig. S3 and Fig. S4. 


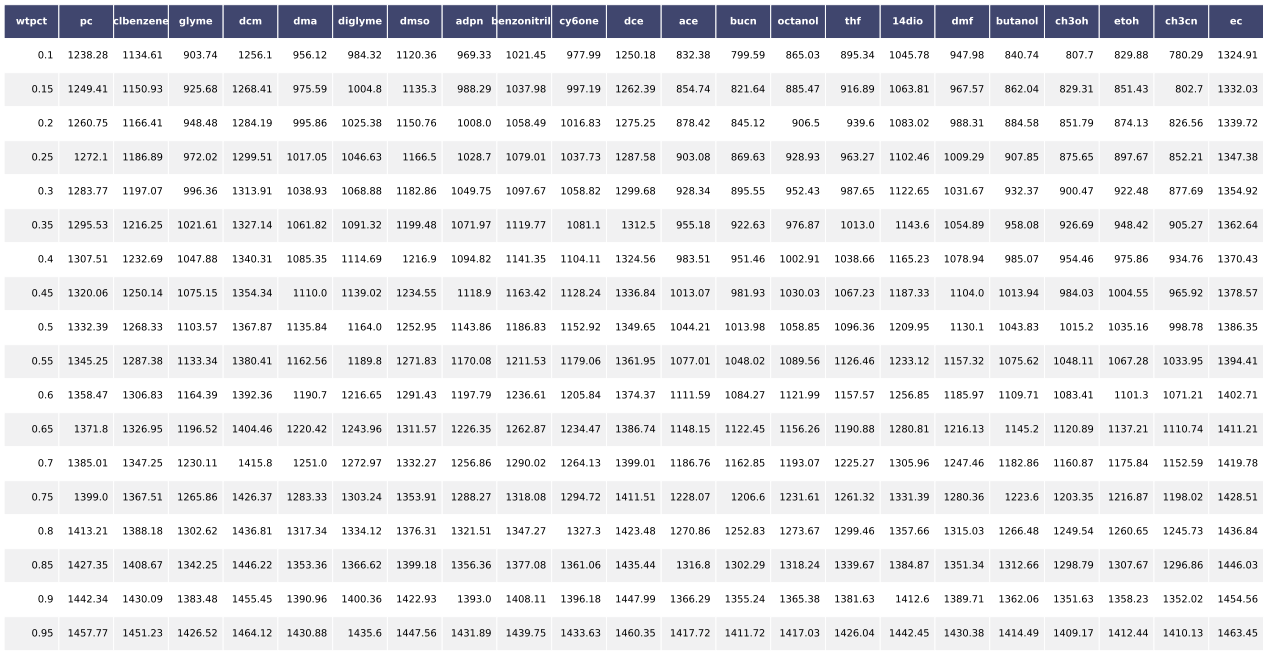

Figure S1: Liquid densities of all mixtures over the range of concentrations in units of mass fraction.

For statistical averaging, the 30 ns trajectory (20,000 frames) is broken up into 750 frame chunks. We have experimented with longer and shorter chunks, but the fits of the stretched exponential functions are not significantly affected.

\section{Dielectric Constants}

The solvent dielectric constants were calculated using the built-in functions in GROMACS. To ensure that these calculations were relatively accurate, we compared the dielectric constants computed from GROMACS to table values, as shown in Fig. S5. The dielectric constants from GROMACS agree relatively well with the table dielectric constants, with several values being underestimated. 


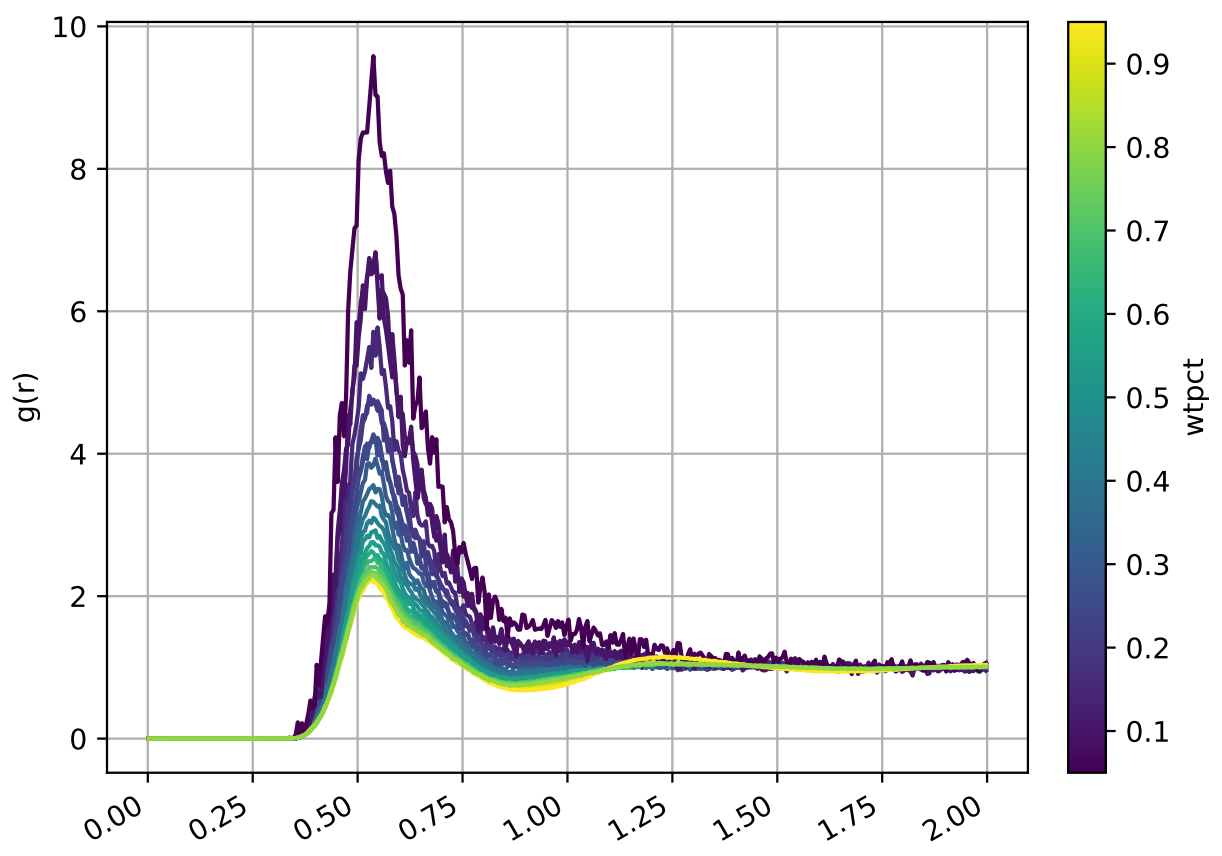

Figure S2: Radial distribution function between $\left[B M I M^{+}\right]$and $\left[\mathrm{Tf}_{2} \mathrm{~N}^{-}\right]$in octanol at various concentrations.

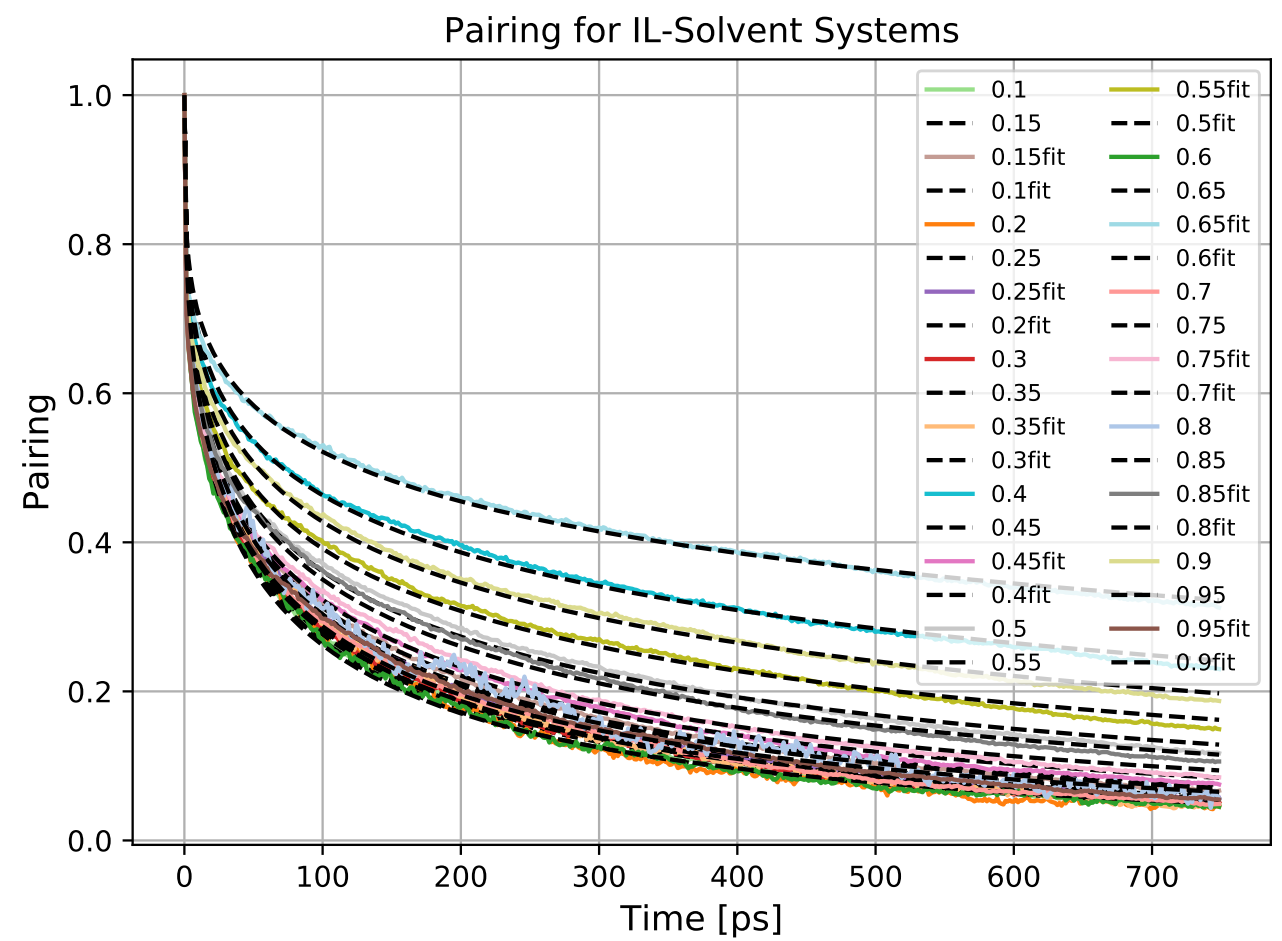

Figure S3: Ratio of paired ions fitted with a stretched exponential function for all $\mathrm{CH}_{3} \mathrm{OH}$ mixtures 


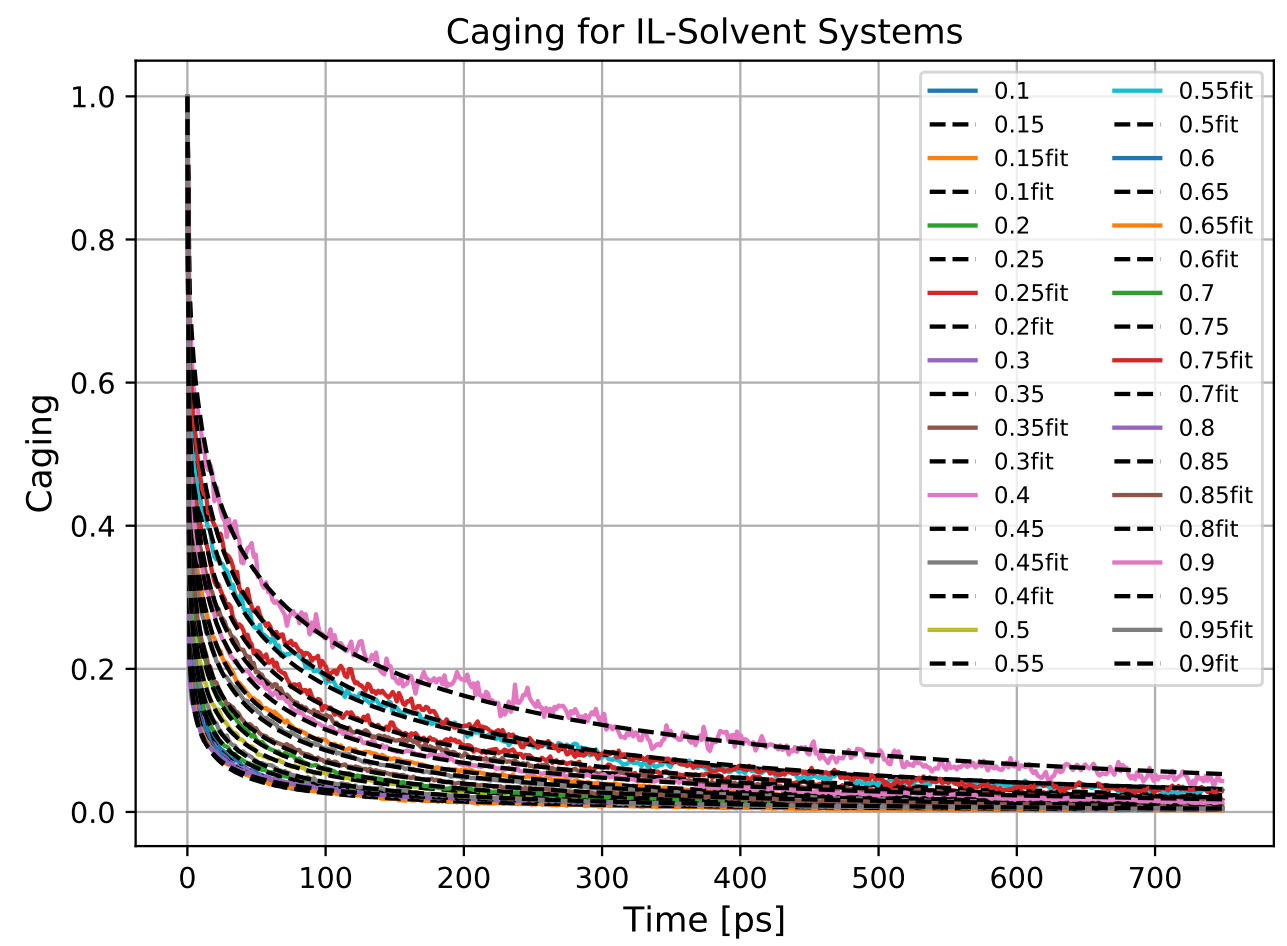

Figure S4: Ratio of caged ions fitted with a stretched exponential function

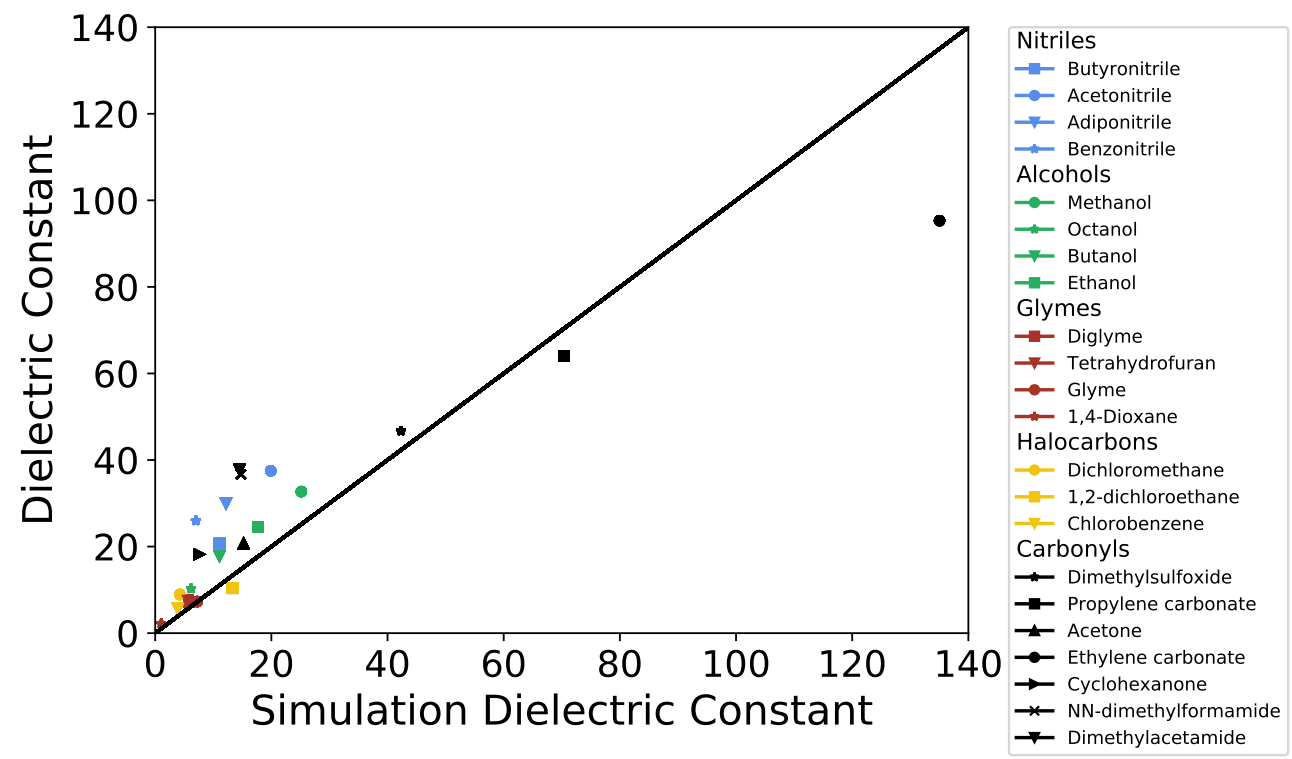

Figure S5: Comparison of dielectric constants computed by simulation and from experimental values. Black line with slope of 1 displayed for comparison purposes. 